\title{
Mappemonde
}

Revue trimestrielle sur l'image géographique et les formes du territoire

\section{Le vignoble d'Azay-le-Rideau (XVIIe-XXIe siècles). À la recherche de facteurs de dynamiques spatiales}

The vineyards of Azay-le-Rideau ( $17^{\text {th }}-21^{\text {st }}$ century). The search for factors of spatial dynamics

Estudio de los factores que influyen en las dinámicas espaciales del viñedo d'Azay-le-Rideau (siglos XVII-XXI)

\section{Adrien Lammoglia et Samuel Leturcq}

\section{(2) OpenEdition}

Journals

Édition électronique

URL : http://journals.openedition.org/mappemonde/2684

ISSN : $1769-7298$

\section{Éditeur}

UMR ESPACE

\section{Référence électronique}

Adrien Lammoglia et Samuel Leturcq, « Le vignoble d'Azay-le-Rideau (XVIle-XXle siècles). À la

recherche de facteurs de dynamiques spatiales », Mappemonde [En ligne], 120 | 2017, mis en ligne le 01 avril 2017, consulté le 12 juin 2020. URL : http://journals.openedition.org/mappemonde/2684

Ce document a été généré automatiquement le 12 juin 2020.

\section{(c) (i) (3)}

La revue Mappemonde est mise à disposition selon les termes de la Licence Creative Commons Attribution - Pas d'Utilisation Commerciale - Partage dans les Mêmes Conditions 4.0 International. 


\section{Le vignoble d'Azay-le-Rideau (XVIIe-XXIe siècles). À la recherche de facteurs de dynamiques spatiales}

The vineyards of Azay-le-Rideau (1 $17^{\text {th }}-21^{\text {st }}$ century). The search for factors of spatial dynamics

Estudio de los factores que influyen en las dinámicas espaciales del viñedo

d'Azay-le-Rideau (siglos XVII-XXI)

Adrien Lammoglia et Samuel Leturcq

\section{Introduction}

En 1959, Roger Dion montre dans son Histoire de la vigne et du vin le poids considérable des facteurs économiques et sociaux dans la répartition de l'activité viticole en France et dans son évolution au cours du temps (Dion, 1959). Les critères géographiques, pédologiques et climatologiques sont pondérés par une approche plaçant le vignoble au cœur d'une conjonction complexe de facteurs liant le contexte « naturel» (sol, plante, climat) au contexte anthropique (économie, société, institutions, culture). La complexité se renforce quand on prend en compte la question du temps dans la répartition actuelle des vignobles, héritage de dynamiques passées. Le temps est volontiers avancé pour expliquer, voire justifier, l'importance ou la faiblesse de vignobles, leur présence ou leur absence dans certaines zones. Le concept de «noyau d'élite» (Kuhnholtz-Lordat, 1963), fréquemment invoqué concernant la viticulture, résulte de cette appréhension. Pourtant, un simple coup d'œil sur l'évolution considérable de la viticulture au cours du dernier siècle doit nous inciter à la prudence. Dans le cadre de cet article, il s'agit d'étudier la dynamique des territoires viticoles et les facteurs qui entrent en jeu, en prenant le cas particulier du vignoble d'Azay-leRideau, en Touraine. 


\section{Le programme VitiTerroir}

2 La question de la dynamique spatiale des vignobles est au cœur du programme VitiTerroir, soutenu par la région Centre-Val de Loire (2014-2016), dont l'ambition est de poser les bases d'une analyse dynamique des territoires viticoles en vue d'élaborer un outil prospectif fondé sur la modélisation des processus de transformation spatiale dans la longue durée (pluriséculaire). Le programme envisage de mener des études historiques de l'activité viticole à plusieurs échelles spatiales (régions, départements, vignobles, parcelles) et temporelles (décennie, demi-siècle, siècle, pluriséculaire), afin de mesurer précisément le poids des facteurs environnementaux et sociétaux dans leur profondeur historique.

\section{Azay-le-Rideau, une commune de Touraine}

3 Le programme VitiTerroir mène en parallèle des études ciblées à diverses échelles temporelles et spatiales permettant d'isoler des conjonctions de facteurs. L'une des fenêtres sélectionnées concerne le vignoble d'Azay-le-Rideau, situé à une vingtaine de kilomètres de Tours. Cette commune tourangelle de 2734 ha est intégrée dans l'Appellation d'Origine Contrôlée (AOC) Touraine. Le territoire de la mention Azay-leRideau, qui comprend 8 communes (Azay-le-Rideau, Rivarennes, Lignières-de-Touraine, Vallères, Artannes-sur-Indre, Thilouze, Saché et Cheillé), est traversé par la vallée de l'Indre, le long de laquelle se concentre actuellement la majeure partie du peuplement ( figure 1).

Figure 1. Situation de I'AOC Touraine-Azay-le-Rideau en Indre-et-Loire

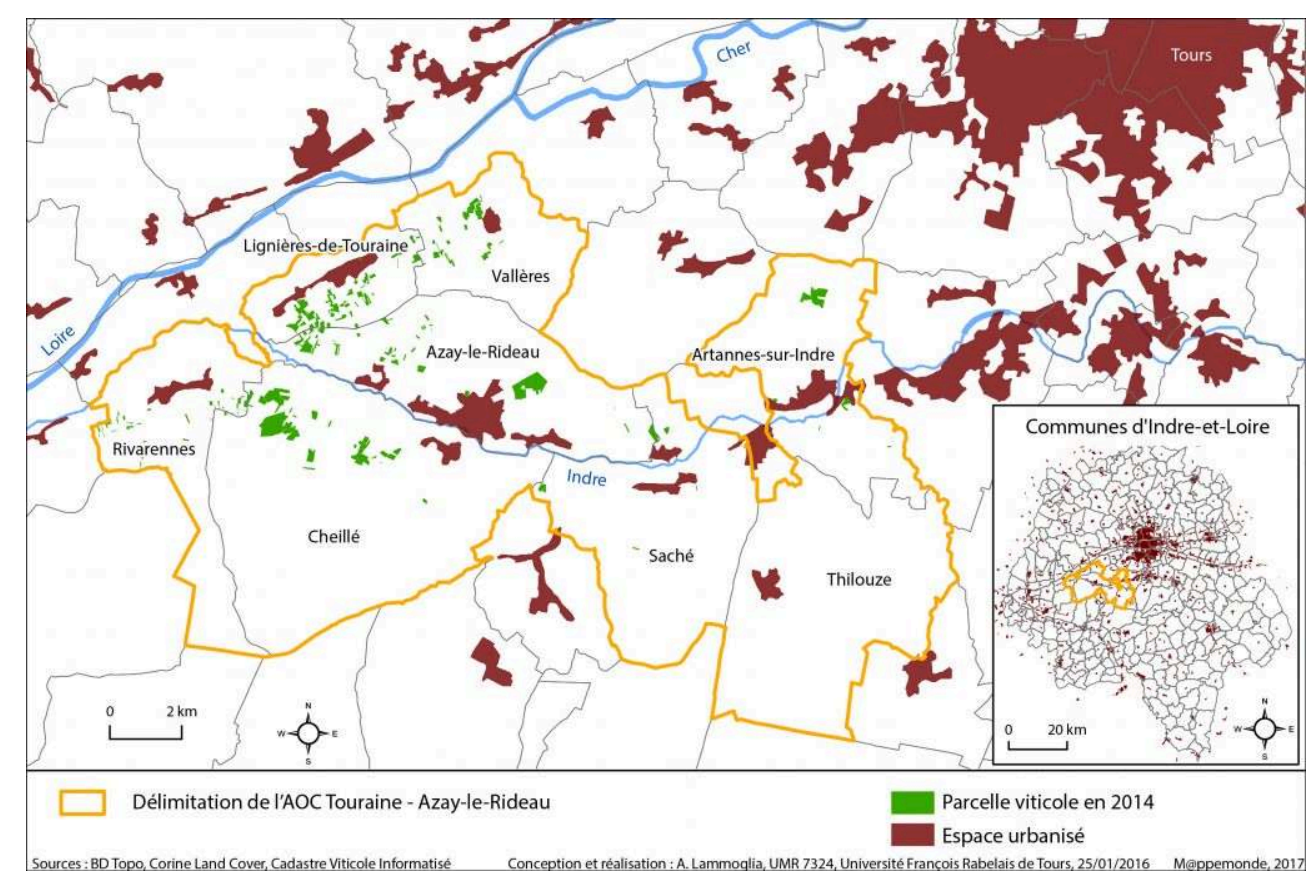

Conception et réalisation : A. Lamoglia, UMR 7324, Université François Rabelais de Tours, 25/01/2016; sources : BD Topo, Corine Land Cover, Cadastre Viticole Informatisé

4 Azay-le-Rideau possède aujourd'hui un vignoble qui, sans avoir la réputation de ceux de Chinon, Bourgueil, Saint-Nicolas-de-Bourgueil, Vouvray ou Montlouis, bénéficie 
toutefois d'une notoriété liée à la présence d'un château, joyau architectural de la Renaissance dans la vallée de la Loire. Mais le choix d'Azay-le-Rideau est avant tout dicté par un impératif documentaire. On dispose en efFet pour cette commune d'un dossier de sources très riches, conservées aux archives départementales d'Indre-etLoire (AD 37) dans le chartrier de la seigneurie d'Azay-le-Rideau, de telle sorte qu'il est possible de reconstituer les grandes lignes de l'évolution spatiale de ce vignoble sur quatre siècles, du début du XVII ${ }^{e}$ siècle à nos jours.

\section{Problématique}

5 Cette analyse historique des dynamiques spatiales du vignoble d'Azay-le-Rideau a pour objectif de classer les facteurs en œuvre dans l'évolution du territoire viticole, afin de formuler une série d'hypothèses quant à leur poids dans le jeu des interactions. À terme, notre ambition est d'identifier les règles d'évolution fondamentales, étape nécessaire dans le travail de modélisation des processus de transformation spatiale. Dans un premier temps, nous mettons en évidence les évolutions spatiales du vignoble ridellois entre le début du XVII ${ }^{e}$ siècle et aujourd'hui. Puis, nous recherchons les éléments d'explication en confrontant les cartes de localisation du vignoble à diverses époques aux données topographiques, pédologiques, démographiques, urbanistiques et réglementaires.

\section{Les sources}

6 Nous avons sélectionné la commune d'Azay-le-Rideau car nous disposons d'une documentation exceptionnellement riche à partir de la seconde moitié du XVe siècle. Les dynamiques spatiales seront analysées à l'échelle de la commune sur une durée de quatre siècles, depuis les années 1610-1635 à 2014.

\section{Phase la plus ancienne : 1610-1635}

7 Le chartrier du seigneur d'Azay, c'est-à-dire les archives de l'administration seigneuriale, renferme des documents détaillant, parcelle par parcelle, la contenance des terres dépendant du seigneur, ainsi que le montant des redevances à verser en échange de leur détention ou de leur exploitation. De fait, ces documents, qui peuvent prendre la forme de déclarations nominatives sur feuillets volants (appelés « aveux et dénombrements ») ou d'une compilation de ces déclarations dans un registre nommé «terrier ", s'apparentent à la documentation cadastrale moderne, si ce n'est qu'il s'agit d'une information de type privé et qu'on ne dispose pas de plan permettant de positionner précisément chaque parcelle dans l'espace paroissial. Ce sont les précisions microtoponymiques qui permettent de localiser approximativement chaque parcelle dans le territoire ridellois. La méthodologie d'utilisation de ces données est explicitée dans Ferreira et al., 2014, p. 208-209.

\section{8-1940 : cadastre napoléonien et statistiques préfectorales}

8 C'est en 1814 que commencent les premières opérations de cadastration du territoire d'Azay-le-Rideau. La levée du plan, en 9 sections, est achevée à la fin du mois de mars 1814. Mais les matrices ne sont réalisées qu'en 1819-1820 (AD 37, 3P144 à 147). Matrices 
et plans permettent de cartographier l'état du vignoble ridellois dans son intégralité en 1820. Il faut toutefois noter qu'il s'agit d'une information ne permettant pas de connaître la densité de l'encépagement.

En 1804 débute la série des enquêtes préfectorales sur les productions agricoles. Regroupées dans la série 6M des AD 37 (6M1289 à 1363), ces archives sont composées de deux types de documents : d'une part, les formulaires adressés à chaque commune, renseignés par les maires et corrigés par les agents voyers cantonaux; d'autre part les tableaux synoptiques par canton réalisés par les agents préfectoraux sur la base des déclarations des maires. Ces statistiques livrent, de 1808 à 1940, une série très complète de données, pour chaque commune du département d'Indre-et-Loire concernant l'état des productions agricoles. Pour la viticulture, nous avons enregistré les superficies viticoles déclarées de 1808 à 1940 avec un pas de temps d'environ 10 ans.

\section{8-2014 : recensement général agricole (RGA) et cadastre viticole}

10 Les données des archives départementales les plus récentes datant de 1940 ont été complétées avec les données du cadastre viticole et dans une moindre mesure avec celles du Recensement Général Agricole (RGA). Le cadastre viticole, qui est informatisé depuis 2010 (CVI), est une base de données exhaustive, enregistrée à la parcelle et entièrement administrée par les services douaniers. Tout viticulteur a le devoir de s'immatriculer et de déclarer chaque année les surfaces encépagées, la quantité de raisin récolté, la production de vin... Grâce au partenariat de VitiTerroir avec l'INAO, il a été possible d'accéder à ces données et d'extraire, sous forme globalisée et anonymisée, les surfaces viticoles par parcelle pour les années 2000, 2005, 2010 et 2014. Nous avons aussi utilisé le cadastre viticole de 1958 qui est un cahier de recensement du vignoble en Indre-et-Loire archivé à l'INAO de Tours. Ce cahier contient entre autres les surfaces plantées par commune (et non pas à la parcelle). Enfin, pour combler le manque d'information entre 1958 et 2000, nous avons utilisé le Recensement Général Agricole (RGA) qui recense les surfaces viticoles à la commune, pour les années 1979, 1988,2000 et 2010.

11 Finalement, il est possible d'établir une cartographie précise de trois phases du vignoble ridellois (figures 2,3 et 4), et de suivre de manière détaillée la progression des surfaces encépagées depuis le début du XIXe siècle (figure 8). Il convient toutefois d'user de prudence dans la comparaison des cartes, car les données ayant servi à les bâtir ne sont pas exactement de la même nature pour chaque période. En efFet, les données du CVI (2014) permettent d'observer la densité des plantations au sein de chaque parcelle, dimension absente des cartes du vignoble en 1820 et 1610-1635. Cet aspect essentiel ne peut donc pas être pris en compte dans l'étude de la dynamique des superficies viticoles. Le CVI et le cadastre napoléonien livrent une information complète, sans aucune lacune. Ce n'est pas le cas des données des années 1610-1635, car il s'agit de sources privées, qui recensent exclusivement les parcelles détenues par le seigneur d'Azay-le-Rideau. Il en résulte une carte lacunaire (figure 4), près de la moitié du territoire n'étant absolument pas renseignée. L'état lacunaire de la documentation oblige à calculer la densité des parcelles viticoles par aire toponymique. C'est de cette manière qu'on repère des zones de plus ou moins forte concentration. 


\section{Analyse historique du vignoble d'Azay-le-Rideau}

12 La dynamique spatiale du vignoble sera observée sur la base d'une cartographie des superficies plantées en vigne à trois moments de son histoire : 2014, 1820, 1610-1635. Nous faisons le choix de partir de l'état le plus récent et le mieux connu pour remonter, de manière régressive, dans les temps plus anciens pour lesquels l'information n'est pas aussi complète. La méthode régressive offre en effet la possibilité d'éclairer la documentation lacunaire des périodes hautes (Leturcq, 2012).

\section{Un petit vignoble relativement concentré au XXI ${ }^{\mathrm{e}}$ siècle}

13 Nous disposons des données les plus récentes (2014) concernant le vignoble ridellois, grâce à la consultation du cadastre viticole informatisé. Ce vignoble couvre aujourd'hui 62,2 ha (soit $2,27 \%$ de la superficie communale), répartis en 89 parcelles, soit une superficie moyenne des parcelles viticoles de 0,69 ha. Cette moyenne doit tenir compte aussi des densités d'encépagement très variables selon les parcelles (figure 2). Le vignoble apparaît comme fortement secondaire dans l'économie rurale de la commune. Son implantation dans l'espace communal se fait exclusivement sur les plateaux, évitant systématiquement la vallée de l'Indre. Si l'on excepte la concentration d'un groupe de grosses parcelles au nord d'Azay-le-Rideau, le long de la route de Tours au lieu-dit « l'Aulée », le reste du vignoble est dispersé, essentiellement dans l'ouest de la commune, dans la continuité du vignoble de la paroisse voisine de Lignières-deTouraine (figure 1).

Figure 2. Surfaces viticoles à Azay-le-Rideau au XXI

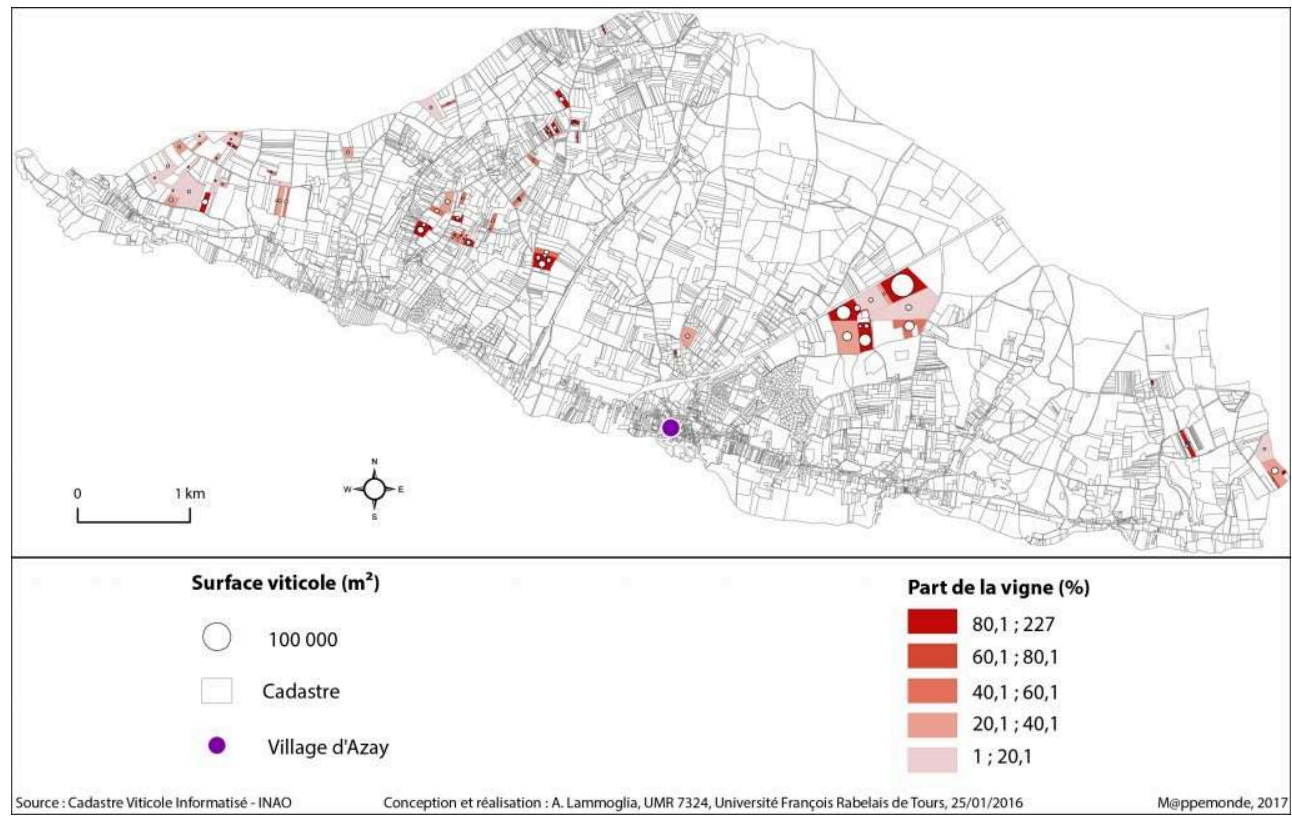

Conception et réalisation : A. Lamoglia, UMR 7324, Université François Rabelais de Tours,

25/01/2016; sources : Cadastre Viticoile Informatisé - INAO 


\section{Un vignoble étendu au $\mathrm{XIX}^{\mathrm{e}}$ siècle} propriétaires différents. La superficie moyenne d'une parcelle de vigne s'élève à 0,16 ha, mais la superficie médiane est moindre, de l'ordre de 0,07 ha. Cette première description quantitative du vignoble ridellois met clairement en évidence l'émiettement poussé des structures de propriété en 1820. La micropropriété domine largement, puisque, en moyenne, chaque propriétaire détient 2,8 parcelles de vigne, avec une médiane à 2 parcelles seulement. Mais en réalité, le vignoble est concentré entre les mains d'une minorité de propriétaires. Si la veuve Cholette ne possède qu'un demi are, Charles de Biencourt, le plus gros propriétaire foncier de la commune, en détient 11,27 ha. 71 propriétaires (soit 6,2\% des détenteurs de parcelles de vigne) possèdent chacun plus de 1,5 ha de vigne, réunissant $118,3 \mathrm{ha}$, soit quasiment $42 \% \mathrm{du}$ vignoble ridellois (Ferreira et al., 2014).

La carte de répartition des parcelles plantées en vigne (figure 3) montre un relatif éclatement du vignoble. Ce qui apparaît le plus nettement, c'est une implantation privilégiée des vignes sur la bordure méridionale de la commune, c'est-à-dire tout le long de la vallée de l'Indre et de la route du fond de vallée, le long de laquelle s'égrainent d'ouest en est des centres de peuplement. À proximité du village d'Azay, où habite une grande partie de la population, se concentre une partie du vignoble. Toutefois, l'essentiel du vignoble ridellois ne se masse pas le long de cette vallée de l'Indre, mais sur un plateau entaillé par deux vallées sèches orientées nord-sud, autour du village de Lionnière, dans le tiers occidental de la commune. C'est là que les vignes sont le plus densément plantées. Enfin, le vignoble s'ancre sur le plateau au nord du village d'Azay-le-Rideau, autour des hameaux de la Fourassière et des Méchinières. Ailleurs, sur les plateaux, ce ne sont que lambeaux de vignoble, petits groupes de parcelles isolées et éparpillées sans logique apparente.

Figure 3. Surfaces viticoles à Azay-le-Rideau au XIXe siècle (1820)

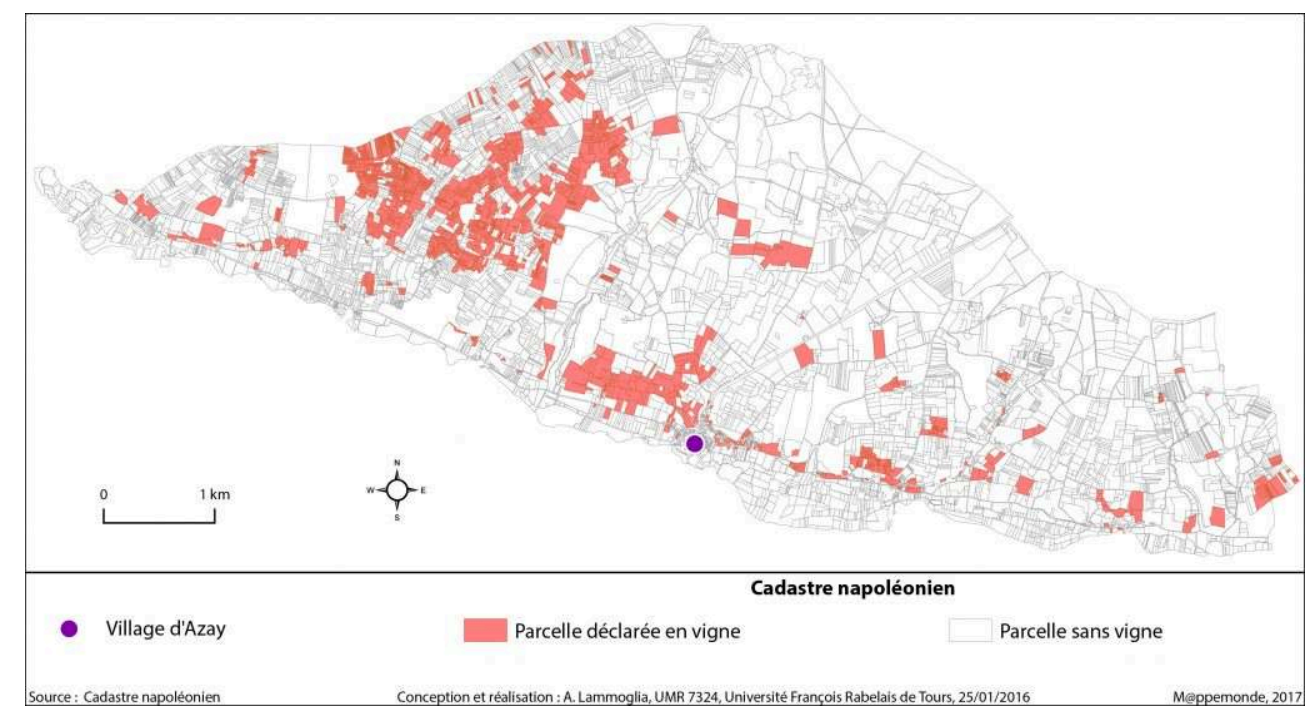

Conception et réalisation : A. Lamoglia, UMR 7324, Université François Rabelais de Tours, 25/01/2016; source : Cadastre napoléonien 


\section{Estimation du vignoble au XVII siècle}

16 $\mathrm{du}$ vignoble ridellois pour la période 1610-1635. Nous nous basons sur la superficie totale de la commune, sur la superficie des lieux-dits renseignés (viticoles et non viticoles) et nous proposons trois scénarios. En premier lieu, nous savons qu'à cette époque la superficie totale du vignoble était comprise entre 62,6 ha (surface connue) et 1 193,2 ha (superficie totale des lieux-dits renseignés). Ensuite, on peut penser que le taux d'occupation des lieux-dits non renseignés était compris entre $5 \%$ (scénario faible) et $20 \%$ (scénario élevé). Cela correspond à une surface comprise entre 119,1 ha et 288,7 ha (tableau 1). Enfin, les aires des lieux-dits viticoles recensées étant occupées en moyenne à hauteur de $12 \%$ par la vigne, si on conserve ce ratio pour les toponymes non renseignés, on peut penser qu'au début du XVII ${ }^{e}$ siècle il y avait environ 195 ha de vigne (scénario moyen). Cette valeur correspond à peu près à la surface recensée en 1808, c'est-à-dire 200 ha (cf. Un vignoble étendu).

Tableau 1. Estimation de la surface viticole totale à Azay-le-Rideau au XVIIe siècle

\begin{tabular}{|l|l|l|}
\hline Estimation & $\begin{array}{l}\text { Surface viticole } \\
\text { totale (ha) }\end{array}$ & Hypothèse \\
\hline $\begin{array}{l}\text { Surface minimale } \\
\text { (connue) }\end{array}$ & 62,6 & seuls les lieux-dits connus possèdent de la vigne \\
\hline Scénario faible & 119,1 & $\begin{array}{l}\text { l'ensemble des lieux-dits non renseignés possède en } \\
\text { moyenne } 5 \% \text { de vigne }\end{array}$ \\
\hline Scénario moyen & 195,2 & $\begin{array}{l}\text { l'ensemble des lieux-dits non renseignés possède en } \\
\text { moyenne } 12 \% \text { de vigne }\end{array}$ \\
\hline Scénario élevé & 288,7 & $\begin{array}{l}\text { l'ensemble des lieux-dits non renseignés possède en } \\
\text { moyenne } 20 \% \text { de vigne }\end{array}$ \\
\hline $\begin{array}{l}\text { Surface maximale } \\
\text { possible }\end{array}$ & 1193,2 & $\begin{array}{l}\text { les lieux-dits de vignes possèdent tous } 100 \% \text { de } \\
\text { vigne }\end{array}$ \\
\hline
\end{tabular}

17 Quoique lacunaire, la documentation met en évidence une situation du vignoble ridellois assurément très difFérente de ce qu'elle est en 1820 (figure 4). À noter que les parcelles en blanc sur la carte ne signifient pas «absence de vigne ", mais «absence d'information ». En premier lieu, on trouve de la vigne en périphérie du village d'Azay et le long de la vallée de l'Indre. On ne trouve aucune trace de la concentration présente en 1820 sur le plateau aux environs du hameau de Lionnière. En revanche, dans certaines parties de la moitié orientale de la commune, le vignoble paraît se concentrer. On repère quatre concentrations :

- dans un secteur immédiatement à proximité de la limite avec la paroisse de Saché, il est possible que ce vignoble s'inscrive dans la continuité du vignoble de Saché ;

- sur le plateau à proximité de la limite paroissiale de Druye ;

- sur le plateau immédiatement au-dessus du hameau de Perré ;

- sur le plateau, immédiatement au nord du village d'Azay-le-Rideau. 
Enfin, il convient de mettre en évidence la présence inattendue de la vigne dans le fond de la vallée, à proximité des rives de l'Indre, exclusivement en zones de prairie en 1820.

Figure 4. Toponymes renseignés et taux d'occupation de la vigne à Azay-le-Rideau au XVII ${ }^{\mathrm{e}}$ siècle (1610-1635)

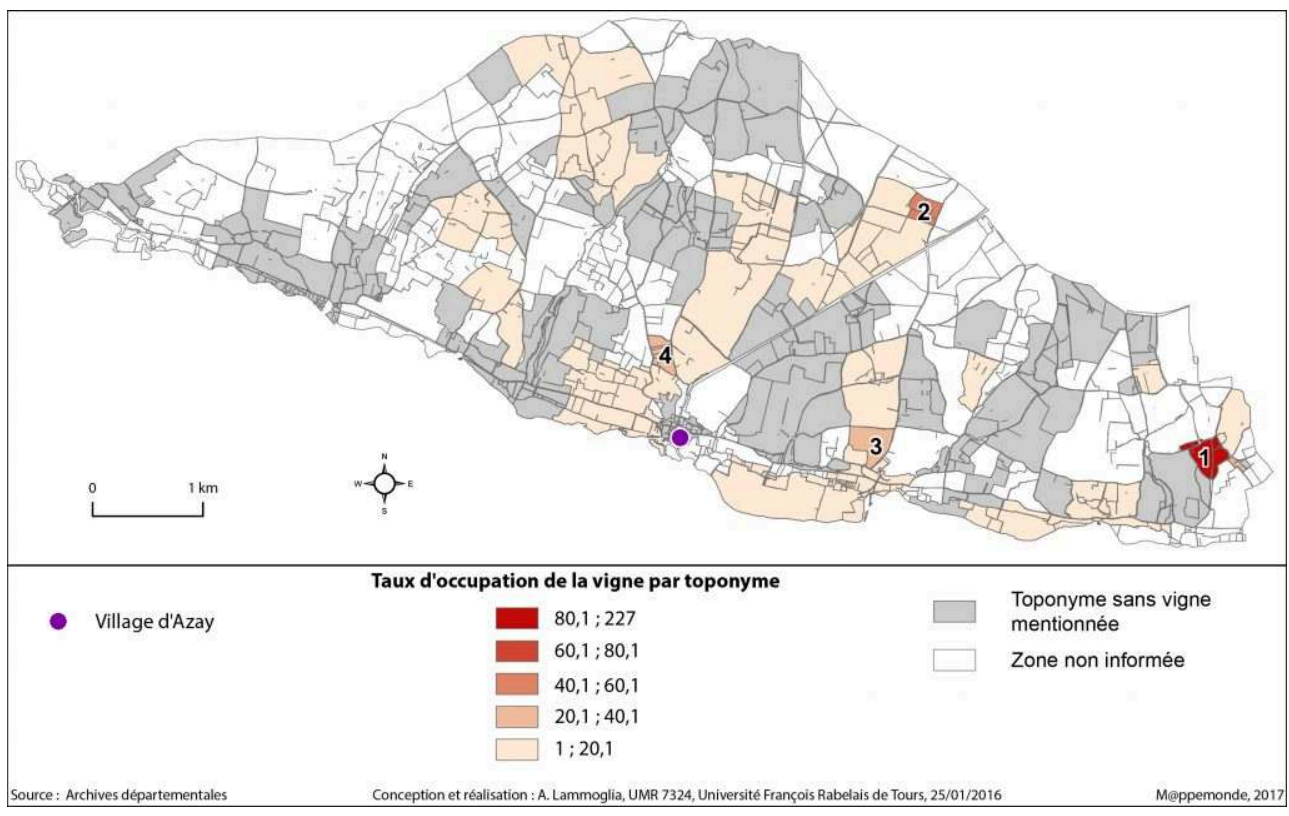

Conception et réalisation : A. Lamoglia, UMR 7324, Université François Rabelais de Tours,

25/01/2016 ; source : Archives départementales

\section{Synthèse des mutations au cours des quatre derniers siècles}

Pour conclure sur la dynamique du vignoble ridellois entre 1610 et 2014, nous proposons une carte de localisation de la vigne pour les trois périodes. Les couches sont alors superposées (figure 5). De par l'hétérogénéité des données (notamment la différence de granularité des données et le manque d'information pour le XVII ${ }^{\mathrm{e}}$ siècle), il n'est pas possible de faire une analyse quantitative des mutations sur les trois périodes. Néanmoins, certains éléments apparaissent très clairement sur la carte. 
Figure 5. Localisation de la vigne à Azay-le-Rideau aux XVII ${ }^{e} X{ }^{e}$ et $X X X^{e}$ siècles

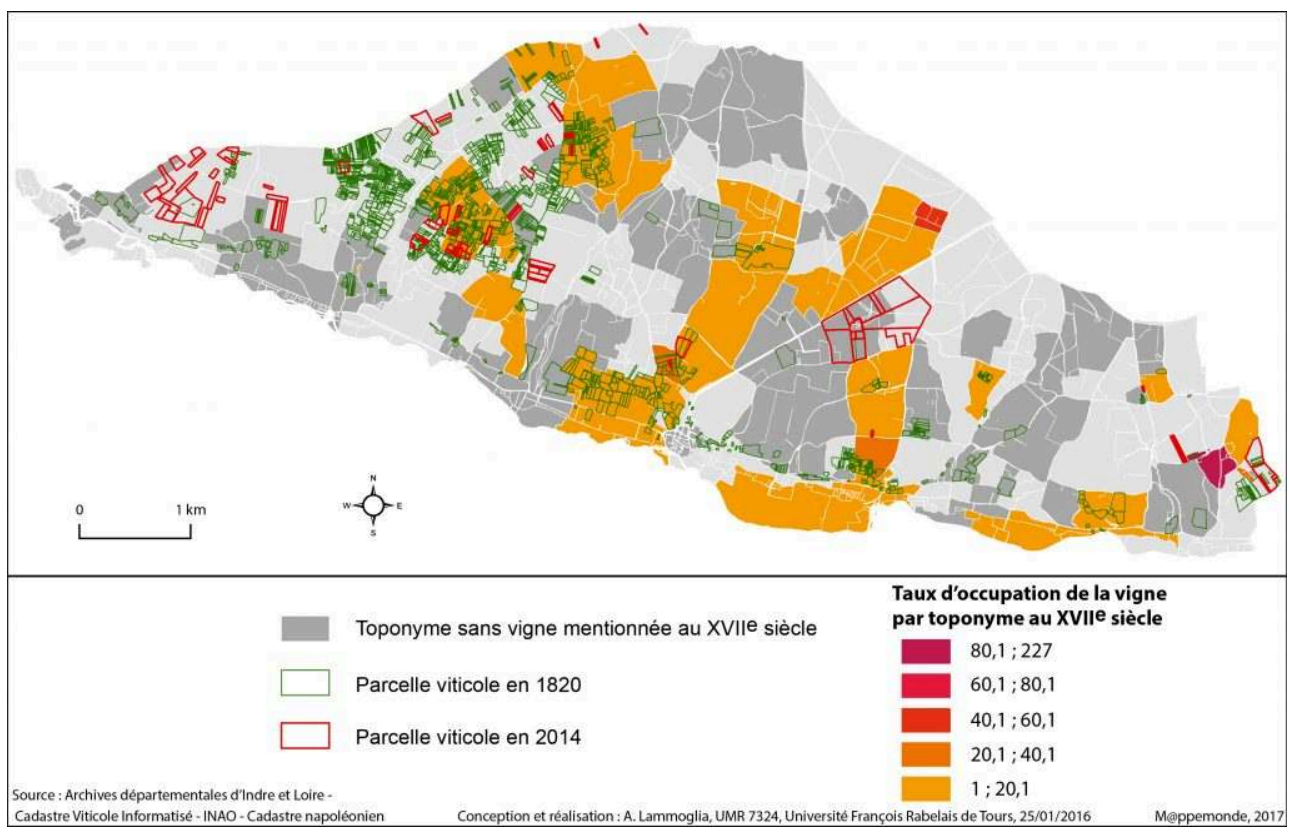

Conception et réalisation : A. Lamoglia, UMR 7324, Université François Rabelais de Tours,

25/01/2016 ; source : Archives départementales d'Indre et Loire - Cadastre Viticole Informatisé -

INAO - Cadastre napoléonien

Tout d'abord, il est intéressant de constater que la forte concentration de la vigne en 2014 au lieu-dit «l'Aulée » n'existait ni en 1820, ni entre 1610 et 1635 . Pourtant, cet espace représente aujourd'hui plus de la moitié du vignoble ridellois ( $38 \mathrm{ha}$ ). La plantation de l'Aulée ${ }^{1}$ le long de la route de Tours s'est faite dans le prolongement des grosses plantations effectuées entre 1820 et 1890, depuis le village d'Azay en s'éloignant de la route (cf. Carte d'état-major). Dans ce cas précis, la route a pu jouer un rôle structurant, et le développement du domaine dans le courant du $\mathrm{XX}^{\mathrm{e}}$ siècle s'inscrit dans la même logique. De la même manière, il semble que la vigne située à l'ouest de la commune, à la frontière avec la commune de Lignières-de-Touraine, soit récente, même si quelques parcelles viticoles étaient bien présentes au XIX ${ }^{\mathrm{e}}$ siècle. On constate ensuite que les deux foyers viticoles du XIX ${ }^{e}$ siècle, à savoir le plateau au nordouest et la partie à l'est (à la frontière avec Saché) ont vu bon nombre de leurs parcelles viticoles disparaitre, converties en parcelles agricoles (notamment des vergers), et dans une moindre mesure en parcelles bâties. Enfin, la quasi-totalité de la vigne le long de l'Indre et à proximité du village d'Azay-le-Rideau a progressivement disparu au cours $\mathrm{du} \mathrm{XX}^{\mathrm{e}}$ siècle pour laisser place à la ville, comme nous le verrons plus bas.

\section{Identification des facteurs en œuvre dans la dynamique spatiale du vignoble}

Les dynamiques spatiales du vignoble ridellois, telles qu'on a pu les mettre en évidence entre le XVII ${ }^{e}$ et le $\mathrm{XXI}^{\mathrm{e}}$ siècle, résultent de la combinaison de plusieurs facteurs. Nous disposons de données précises et fiables permettant d'examiner l'influence de cinq d'entre eux : topographie, pédologie, démographie, urbanisation et labellisation (en l'occurrence seulement l'impact du classement en Appellation d'Origine Contrôlée). 


\section{Le facteur topographique}

La commune d'Azay-le-Rideau se situe à l'aval de la vallée de l'Indre. Les pentes les plus fortes du coteau le long de l'Indre sont comprises entre 15 et $33 \%$ et on observe une zone de faible pente $(<5 \%)$ à l'ouest de la commune. L'altitude moyenne du fond de vallée est de $40 \mathrm{~m}$ et elle avoisine $85 \mathrm{~m}$ sur les plateaux. Bien que le village d'Azay-leRideau s'étire le long de l'Indre, l'essentiel de la surface communale se situe sur les plateaux $(73,3 \%)$. Trois ruisseaux orientés nord-est sud-ouest viennent découper ces plateaux créant ainsi des petites vallées de fortes pentes (15 à $30 \%$ ) (figure 6).

Figure 6. Pentes et localisation de la vigne à Azay-le-Rideau aux XIX et XXIe siècles

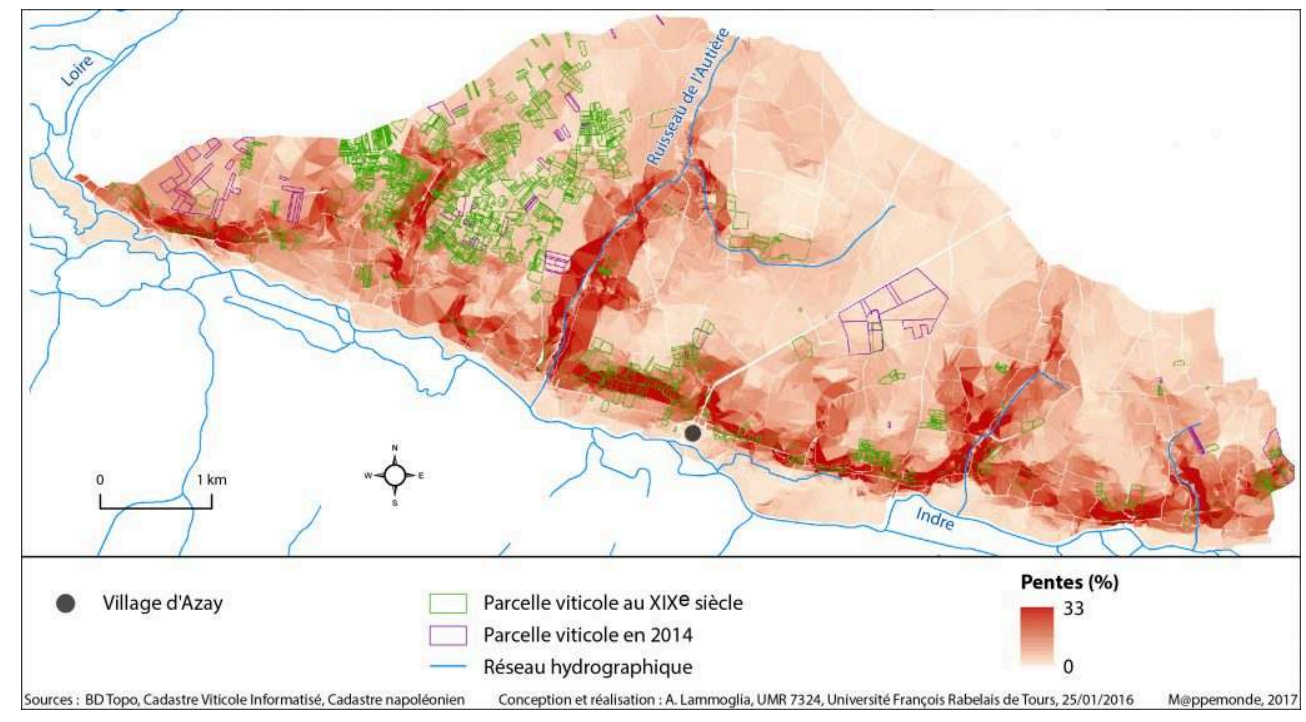

Conception et réalisation : A. Lamoglia, UMR 7324, Université François Rabelais de Tours, 25/01/2016 ; source : BD Topo, Cadastre Viticole Informatisé, Cadastre Napoléonien

En 1820 le vignoble ridellois est situé sur deux types de terrains viticoles : d'une part, les coteaux à proximité du village; d'autre part, le plateau au nord-ouest de la commune. Quasiment les deux tiers du vignoble (58\%) se trouvaient sur des pentes inférieures à $5 \%$, et un tiers $(32,5 \%)$ sur des pentes comprises entre 5 et $15 \%$ (Ferreira, 2009). Le vignoble d'Azay-le-Rideau est donc en 1820 un vignoble de faible pente, voire de plateau (près de $80 \%$ du vignoble). Le constat est encore plus vrai au XXI ${ }^{\mathrm{e}}$ siècle puisque l'on constate que les vignes situées sur les coteaux ont toutes disparu; le vignoble est aujourd'hui principalement sur les plateaux (figure 6). La comparaison avec la situation des terrains viticoles en 1610-1635 est d'autant plus intéressante qu'elle confirme ce constat. Le vignoble est préférentiellement installé sur les plateaux, mais avec un trait original totalement absent du XIX ${ }^{e}$ au XXI ${ }^{e}$ siècle : la présence de vignes dans les zones basses humides de la vallée de l'Indre.

L'analyse des dynamiques spatiales dans la longue durée nous montre qu'il est possible de cultiver la vigne aussi bien sur les plateaux, sur les coteaux (même les plus abrupts), que dans les fonds de vallée. Néanmoins, les faibles pentes, voire les terrains plats, sont préférées aux zones fortement pentues. On observe aussi, depuis le XVII ${ }^{e}$ siècle, une tendance du vignoble à abandonner les zones basses. 


\section{Le facteur pédologique}

La qualité du sol est une donnée essentielle pour comprendre l'implantation actuelle des vignobles. L'était-ce autant au cours des périodes plus anciennes? L'étude de ce facteur repose sur l'analyse de deux documents complémentaires :

- la carte des sols réalisée par la Chambre d'Agriculture d'Indre-et-Loire, dont la résolution est le $1 / 50000$ et qui ofFre une information sur l'ensemble de la commune d'Azay-le-Rideau ( figure 7) ;

- la cartographie des unités de terroir à l'échelle du 1/5000 ou 1/10 000 réalisée par la Cellule Terroir Viticole et librement consultable sur le site e-terroir. Ce document très précis livre cependant une information limitée aux seuls secteurs en AOC, soit un tiers seulement du territoire ridellois.

Ces données ont été analysées et discutées avec François Garnotel (ingénieur agronome de l'INAO Tours) et Sébastien Salvador-Blanes (Maître de conférences de l'université François-Rabelais, pédologue de l'équipe GéhCo), qui ont participé à la révision récente de la délimitation AOC à Azay-le-Rideau.

Figure 7. Sols et localisation de la vigne à Azay-le-Rideau aux XIXeet XXIe siècles

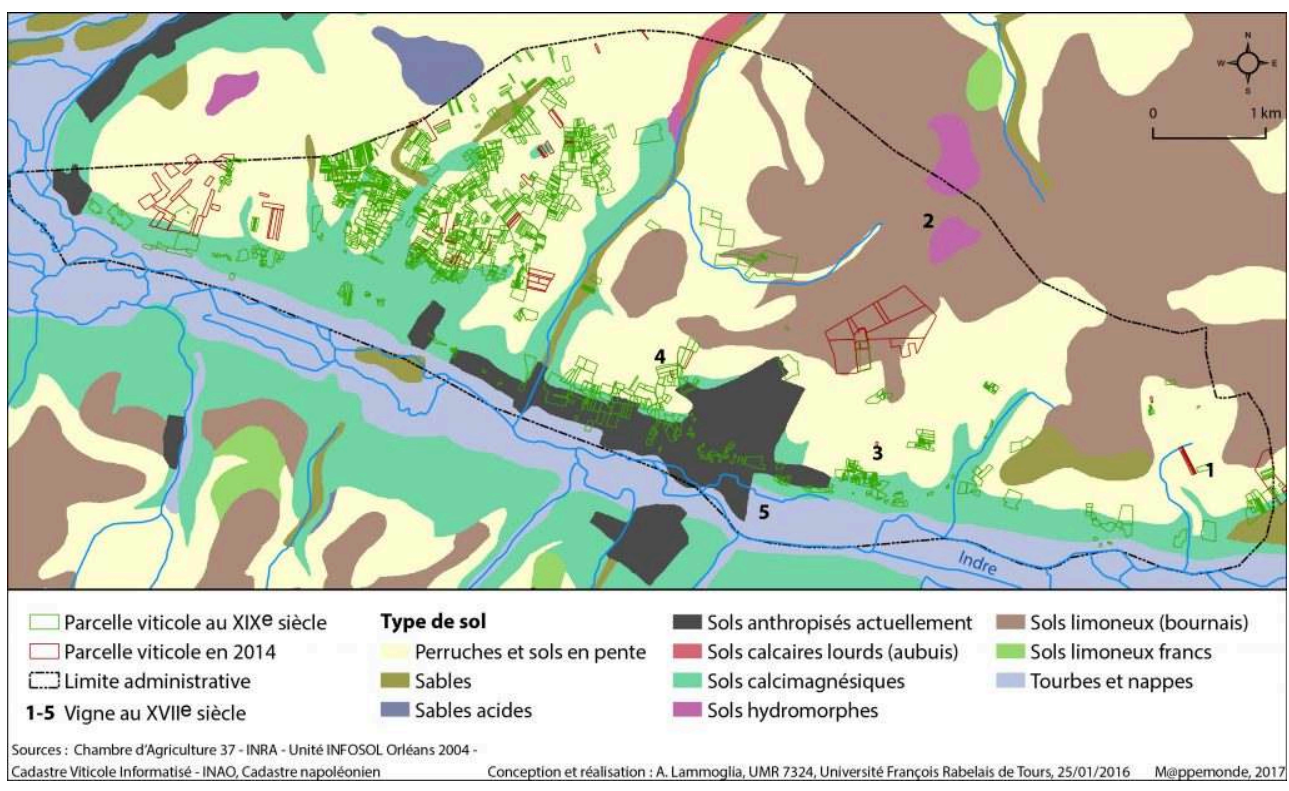

Conception et réalisation : A. Lamoglia, UMR 7324, Université François Rabelais de Tours, 25/01/2016 ; source : Chambre d'agriculture 37 - INRA - Unité INFOSOL Orléans 2004 - Cadastre Viticole Informatisé - INAO, Cadastre napoléonien

En 1820, l'essentiel du vignoble est localisé sur les sols de perruches, dénomination spécifiquement tourangelle des sols caillouteux argileux, aujourd'hui considérés comme des sols propices à l'activité viticole. Il convient toutefois de souligner que, d'une part, le vignoble se concentre sur un seul secteur des perruches (plateau nord-ouest) et que, d'autre part, la vigne s'implante aussi sur les sols calcaires dans la vallée de l'Indre, peu propices à la viticulture. Il en résulte qu'au XIX siècle, la qualité des sols n'est une condition ni nécessaire ni suffisante pour expliquer la présence ou l'absence de viticulture dans un secteur. 
28 Actuellement, le vignoble est principalement installé sur les sols de perruches, évitant les sols calcaires. Cependant, le domaine de l'Aulée (au nord du village d'Azay le long de la route de Tours) est situé sur un sol limoneux, généralement mal drainé (appelé «bournais » en Touraine), c'est-à-dire un sol a priori peu favorable à la viticulture. Cette implantation, non conforme aux prescriptions habituelles des délimiteurs, tient à la présence du petit manoir de l'Aulée autour duquel un domaine s'est reconstruit dans la période post-phylloxérique, et certainement à la route de Tours qui offre une exposition commerciale intéressante.

Les données disponibles pour le vignoble ridellois du XVII ${ }^{e}$ siècle montrent une localisation sur les sols de perruches (concentrations 3 et 4 sur la figure 7). Mais cette implantation n'est pas exclusive. En efret, le vignoble sis aux confins de la forêt de Villandry (2) est localisé sur des sols de bournais, en limite d'une poche de sol clairement hydromorphe. En outre, des vignes existent dans le fond de vallée, au bord de l'Indre (5). Il convient enfin de remarquer que le vignoble sis en limite de la paroisse de Saché (1) est planté sur des sols de perruches, mais aussi peut-être partiellement sur des sols calcaires lourds (appelé " aubuis ») ; la résolution de la carte des sols n'est pas suFfisante pour trancher cette question.

30 À l'issue de cette analyse, on constate une permanence: les perruches, aujourd'hui réputés favorables pour la viticulture, sont privilégiés pour l'implantation du vignoble $\mathrm{du} \mathrm{XVII}{ }^{\mathrm{e}}$ au XXI ${ }^{\mathrm{e}}$ siècle. Mais la plantation des vignes ne se fait pas exclusivement sur ce sol. Dans la période préphylloxérique, la viticulture se développe aussi sur des sols aujourd'hui considérés comme peu propices (bournais), voire carrément néfastes (sols calcaires et tourbeux). Il résulte de ces observations que, pour comprendre les dynamiques historiques de l'implantation de la vigne, le sol est un facteur certes important, mais qu'il ne suffit pas à expliquer la présence ou l'absence de vigne pour chaque époque. Des facteurs sociologiques, culturels, géographiques, réglementaires... sont à rechercher.

\section{Le facteur démographique}

31 La démographie est un facteur essentiel à analyser, car elle conditionne à la fois la production et la consommation, pouvant impacter de ce fait très directement les surfaces viticoles. Cette donnée est aisément accessible grâce à l'important travail de dépouillement des archives mené par le Laboratoire de démographie historique (LDH) de l'EHESS. Cette équipe a constitué une base de données de la démographie des communes du XVII siècle à nos jours, en compilant les données de la série des Dictionnaires des paroisses et communes de France (Gorry, 2007). Cette base, accessible en ligne, ofFre la possibilité de croiser la dynamique démographique ridelloise avec celle des surfaces du vignoble pour une période de temps comparable (figure 8). La relation entre les deux jeux de données a pu être mise en évidence par un coefficient de corrélation pour trois périodes, clairement identifiables sur la courbe des superficies viticoles : 1687-1882 (10 points), 1882-1932 (12 points), 1932-2012 (12 points). 
Figure 8. Évolution de la population et des surfaces viticoles à Azay-le-Rideau entre 1687 et 2014

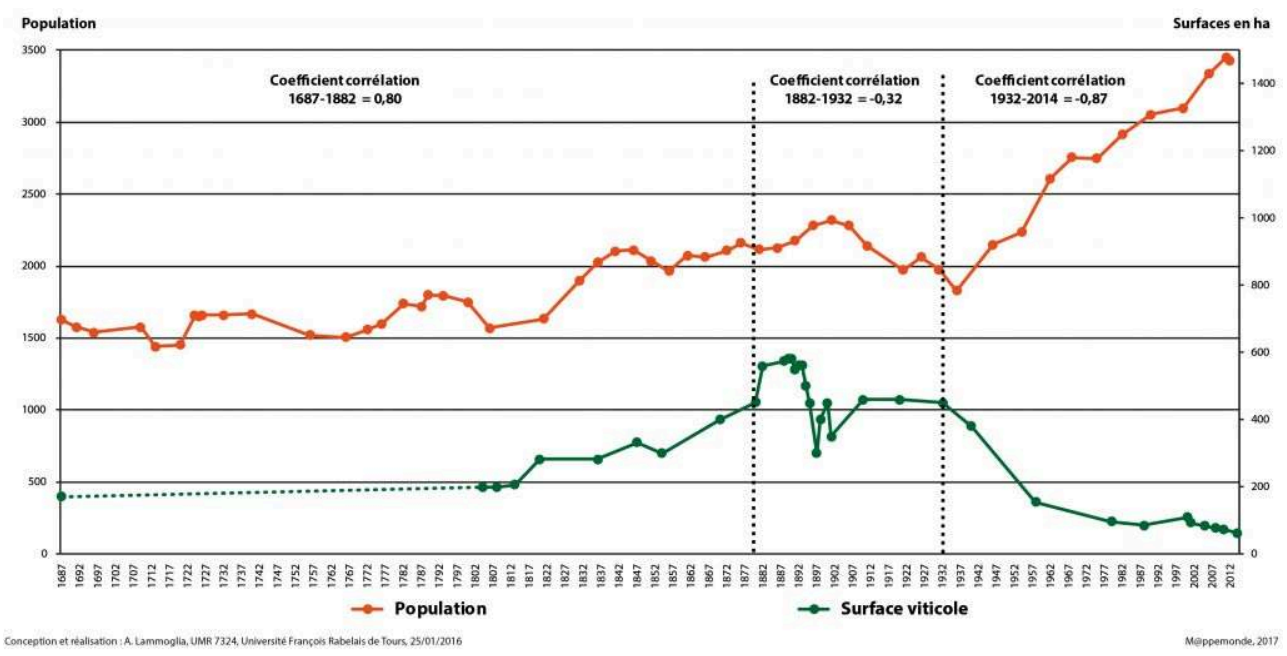

Conception et réalisation : A. Lamoglia, UMR 7324, Université François Rabelais de Tours, 25/01/2016

Pour la période 1687-1882, la corrélation positive entre la croissance démographique et la croissance des surfaces en vigne est remarquable, mettant en évidence un lien fort entre le peuplement et l'encépagement qui croissent de manière régulière. À partir de 1882 débute une période de totale décorrélation. Le début des années 1880 marque en efFet le début du phylloxéra en Touraine, dont les attaques ont entraîné une déstructuration du vignoble (Garrier, 1989 ; Tessier, 2010). Les oscillations de la courbe des surfaces viticoles de 1880 à 1907 témoignent de cette crise. Paradoxalement, elle ne se manifeste pas par un efFondrement des superficies plantées, mais par une crue forte et subite des encépagements (passant de 451 ha en 1880 à 560 ha en 1882, pour culminer à 580 ha en 1889/1890). À partir de 1891, on observe une série d'eFFondrements et de rattrapages partiels, qui donnent à la courbe cet aspect fluctuant caractéristique jusqu'aux premières années du $\mathrm{XX}^{\mathrm{e}}$ siècle. La superficie viticole se stabilise alors jusqu'aux années 1930 à 450/460 ha, alors qu'au même moment la commune d'Azay-le-Rideau connaît une décrue assez sensible de sa population. C'est dans le courant des années 1930 que s'amorce le déclin de la viticulture ridelloise, alors que la population d'Azay amorce une forte croissance démographique. La corrélation négative est alors très marquée.

Ce graphique est intéressant en ce qu'il montre la succession de deux phases fondamentales autour de la charnière de la crise du phylloxéra. Une première période, qu'on pourrait qualifier de "traditionnelle", met en évidence une viticulture artisanale dédiée à l'autoconsommation et au marché local. La croissance des surfaces viticoles est à mettre en lien avec la croissance démographique. La seconde période, post-phylloxérique, met en exergue une évolution inverse des superficies viticoles et de la population. Des phénomènes économiques et sociaux semblent en œuvre, comme l'abandon de la pratique de l'autoconsommation, la modification des goûts, la baisse de la consommation...

\section{Urbanisation}

Le tableau 2 permet de porter un regard croisé sur les tendances démographiques, urbaines et viticoles. On constate qu'entre 1820 et 2012, la population d'Azay-le-Rideau 
a été multipliée par 2, ce qui a entraîné un accroissement des parcelles bâties (multipliées par 6,4). Les surfaces viticoles, quant à elles, ont été divisées par 4,5. Ainsi, on constate une prédominance des parcelles viticoles ( $282 \mathrm{ha}$ ) par rapport au bâti (63 ha) en 1820 et une tendance inverse en 2012 (62,2 ha de vigne contre 406,64 ha de parcelles bâties). En deux siècles, il y a donc bien eu un changement très fort en termes d'occupation des sols.

Tableau 2. Évolution de la démographie, des surfaces bâties et viticoles au cours des deux derniers siècles

\begin{tabular}{|l|l|l|}
\hline & 1820 & 2012 \\
\hline Population (hab.) & 1634 & $3431(+110 \%)$ \\
\hline Surfaces bâties (ha) & 63 & $406,64(+545 \%)$ \\
\hline Surfaces viticoles (ha) & 28262,2 & $(-77 \%)$ \\
\hline
\end{tabular}

Dans une approche plus spatiale, on observe au XIX ${ }^{\mathrm{e}}$ siècle une structure atomisée du peuplement (figure 9). Mis à part le village d'Azay-le-Rideau qui demeure le centre urbain, la répartition des foyers de peuplement est relativement homogène sur l'ensemble du territoire communal. On trouve également de la vigne un peu partout (à part dans le nord-est) avec une forte concentration sur les coteaux et sur le plateau au nord-ouest.

Figure 9. Bâti et parcelles viticoles à Azay-le-Rideau au début du XIXe siècle

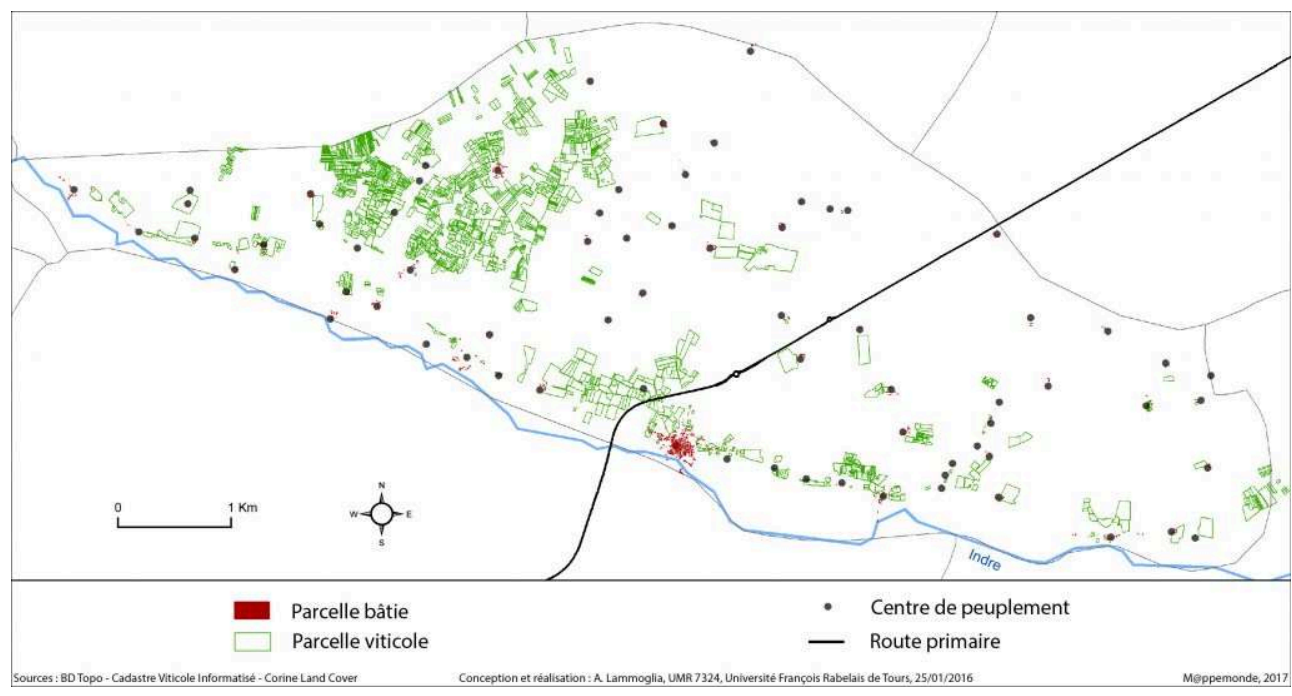

Conception et réalisation : A. Lamoglia, UMR 7324, Université François Rabelais de Tours, 25/01/2016 ; sources : BD Topo - Cadastre Viticole Informatisé - Corine Land Cover

En revanche, au XXI ${ }^{\mathrm{e}}$ siècle, la morphologie urbaine est sensiblement difFérente ( figure 10). Le cœur de ville s'est densifié et le périurbain s'est étalé, principalement le long de la vallée, de la route nationale et sur le plateau au nord-ouest. Concernant le vignoble, une grande part des vignes situées sur le plateau a disparu. Certaines parcelles ont été bâties et d'autres sont toujours agricoles, mais reconverties 
principalement en vergers selon les données du Registre Parcellaire Graphique (RPG) de 2012. La vigne qui se situait à proximité du village d'Azay au XIX ${ }^{\mathrm{e}}$ siècle, notamment sur les coteaux, a disparu, hormis deux parcelles toujours en exploitation en 2014. On constate aussi que des parcelles de grande taille ont été encépagées dans le prolongement nord de la ville, le long de la route nationale (domaine de l'Aulée).

Figure 10. Bâti et parcelles viticoles à Azay-le-Rideau au XXIe siècle

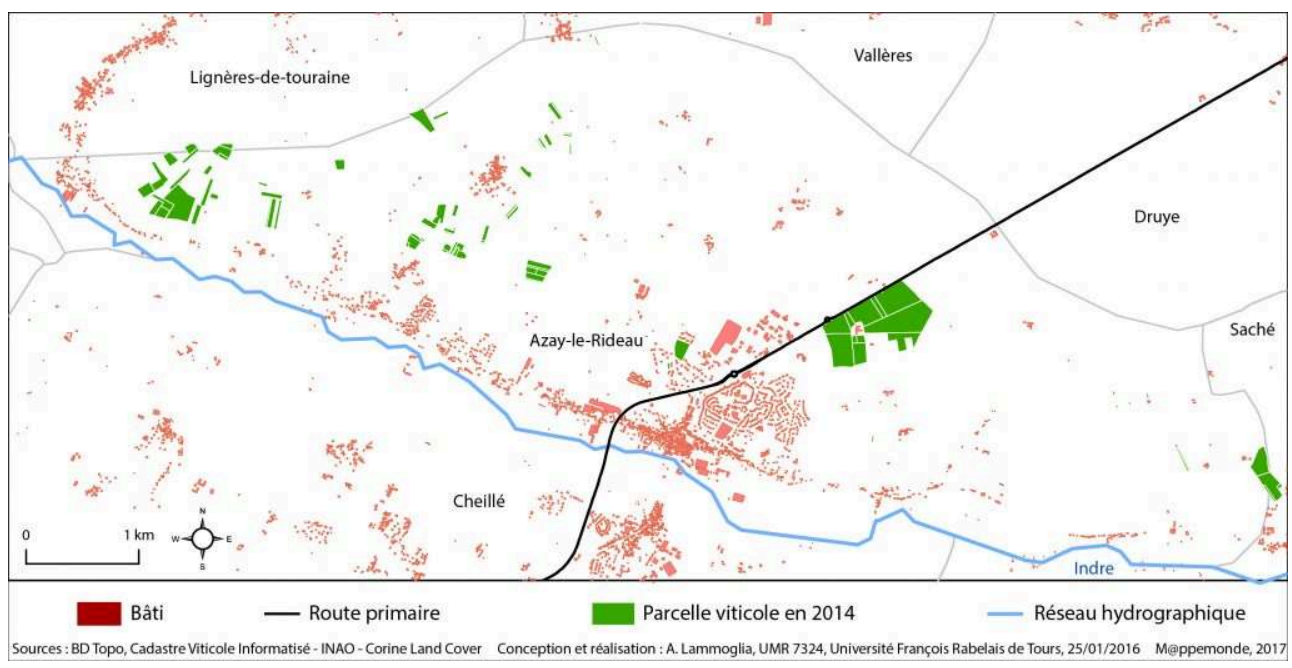

Conception et réalisation : A. Lamoglia, UMR 7324, Université François Rabelais de Tours, 25/01/2016; sources : BD Topo - Cadastre Viticole Informatisé - INAO - Corine Land Cover

La figure 11 est un gros plan sur le village d'Azay-le-Rideau. La superposition des quatre couches (surface bâtie et surfaces viticoles pour les années 1820 et 2012) permet d'analyser finement l'évolution des parcelles encépagées et urbaines. Il apparaît que l'essentiel des surfaces bâties contemporaines ne se sont pas développées sur les terres viticoles du XIX siècle. En efFet, on observe un partitionnement assez net de l'occupation du sol avec, sur la partie est et nord-est, l'essentiel des surfaces bâties contemporaines et sur la partie ouest les surfaces viticoles du XIX ${ }^{e}$ siècle qui n'ont été que partiellement bâties. On y trouve aujourd'hui surtout des prairies, des vergers et quelques grandes cultures. En somme, cette carte montre que le mitage urbain n'est que partiellement responsable de la rétraction des surfaces viticoles au niveau du village d'Azay-le-Rideau. 
Figure 11. Bâti et parcelles viticoles aux $\mathrm{XIX}^{\mathrm{e}}$ et $\mathrm{XXI^{e }}$ siècles (zoom sur le cœur de ville)

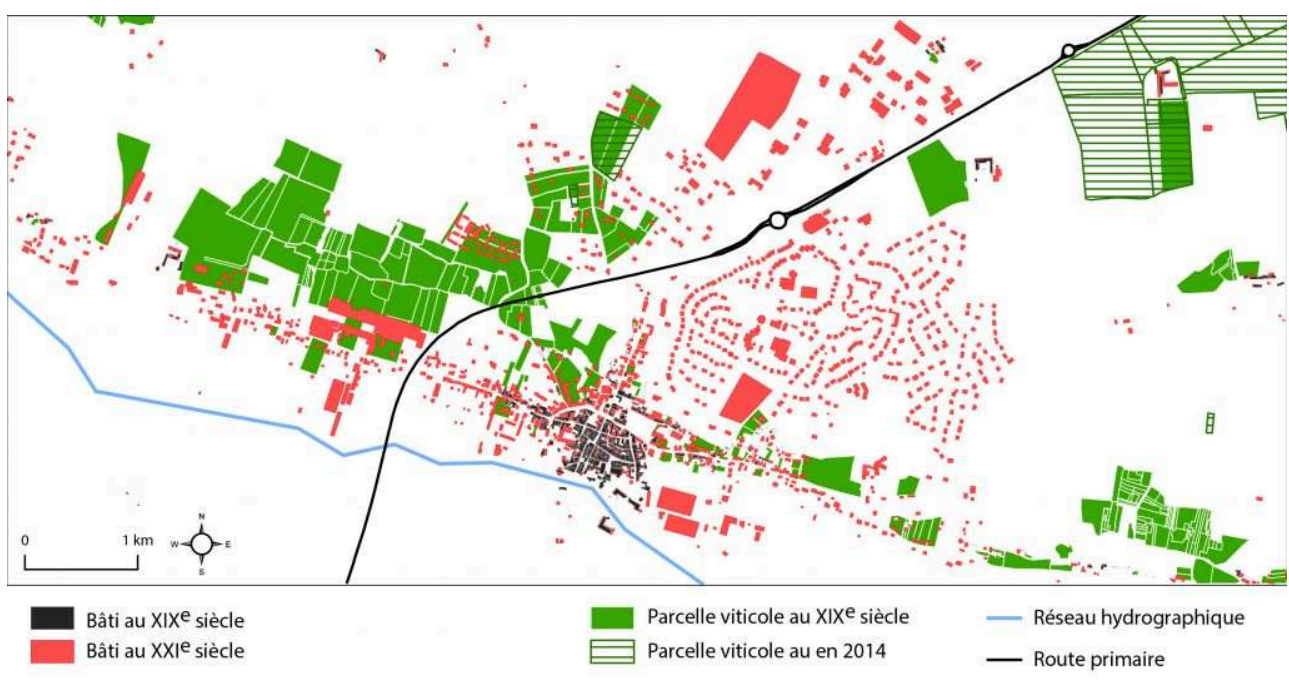

Conception et réalisation : A. Lamoglia, UMR 7324, Université François Rabelais de Tours,

25/01/2016; sources : BD Topo - Cadastre Viticole Informatisé - INAO - Corine Land Cover

38 Finalement, cette analyse réalisée à une échelle très fine montre que la compétition spatiale entre l'urbain et la vigne est relativement faible, malgré la relation statistique inverse entre la population et les surfaces viticoles. Il ne s'agit donc pas d'une relation linéaire de cause à efFet, mais bien d'une superposition et d'une mise en interaction de plusieurs facteurs à la fois démographique, sociologique, économique, politique...

\section{Impact de la labellisation AOC}

Le poids de la réglementation dans la structuration des vignobles est complexe, car si les textes réglementaires visent à conformer des activités à une volonté politique normative en développant des mesures incitatives ou contraignantes, l'application de la réglementation n'est pas nécessairement efFective, ou les efFets attendus ne sont pas forcément visibles. Le facteur réglementaire doit être pris en considération avec beaucoup de prudence. D'autant plus qu'avec la mondialisation des échanges, les vignobles sont soumis à une réglementation à plusieurs étages, depuis les accords internationaux (OMC), la réglementation européenne (PAC, discussion sur la libéralisation des droits de plantation) et leur application au niveau national. Nous nous contenterons d'une approche locale réduite à la question de l'impact de la labellisation AOC à partir des années 1930.

À la suite de la crise du phylloxéra, la reconstruction du vignoble et de l'économie viticole ont reposé sur une politique de labellisation des productions, en vue de garantir la qualité et de lutter contre les fraudes. Le vignoble d'Azay-le-Rideau a été concerné par cette politique. Le 24 décembre 1939 est délivrée l'AOC Touraine, dans laquelle vient s'intégrer le vignoble ridellois. À la différence de Chinon, Vouvray, Saint-Nicolas-de-Bourgueil et Bourgueil, Azay-le-Rideau ne parvient pas à se voir reconnaître une appellation qui lui soit propre. Azay obtient toutefois en 1953 une mention dans l'AOC Touraine pour les vins blancs, complétée en 1976 par une mention pour les vins rosés. La délimitation a été reprise et afFInée en 2010. Est retenu dans 
cette aire de délimitation l'ensemble des parcelles qui étaient plantées à la veille de la crise du phylloxéra et au moment des opérations de délimitation dans les années 1930.

La corrélation de la dynamique spatiale du vignoble ridellois avec la mise en place de la labellisation $\mathrm{AOC}$ n'est pas évidente à analyser car ce facteur intervient et interagit sans aucun doute avec :

- la forte croissance démographique que connaît dans le même temps Azay (cf. Le facteur démographique) ;

- la progression de l'urbanisation qui se fait partiellement au détriment des superficies viticoles (cf. Urbanisation);

- la forte baisse de la consommation et l'évolution des goûts ;

- la concurrence des appellations voisines de Chinon, Bourgueil et Saint-Nicolas-de-Bourgueil.

Il convient aussi de comparer la progression ridelloise avec celle des autres communes de la mention Azay-le-Rideau de l'AOC Touraine (figure 12). Si l'on omet le cas exceptionnel de Lignières-de-Touraine, l'ensemble des communes connait une décroissance de la superficie plantée, avant la mise en place de la labellisation AOC Touraine en 1939, alors que se manifeste dans le même temps une croissance démographique dès 1936. Cette évolution met en évidence un relatif échec de la politique de labellisation, dans la mesure où elle n'est pas parvenue à enrayer la déprise viticole et la reconversion des sols, en partie en sol bâti (cf. Urbanisation) et en vergers. Ce mode de reconversion montre le poids du facteur économique, mais aussi sociologique. Manifestement, la viticulture n'assurait plus une rentabilité suFfisante au regard de l'évolution des modes de consommation (abandon de l'autoconsommation, évolution des goûts). Cela résulte sans doute aussi de la capacité des vignerons à mettre en avant leurs productions, face à des appellations mieux organisées et structurées, développant une véritable politique de communication commerciale. Face à la concurrence d'appellations ayant su valoriser leur label AOC (Saumur-Champigny, Chinon, Bourgueil, Saint-Nicolas-de-Bourgueil), le vignoble ridellois s'est réduit et semble se concentrer entre les mains d'une petite élite en capacité de développer une visibilité commerciale.

Figure 12. Évolution des surfaces viticoles par communes de l'AOC Touraine-Azay-le-Rideau entre 1910 et 2014

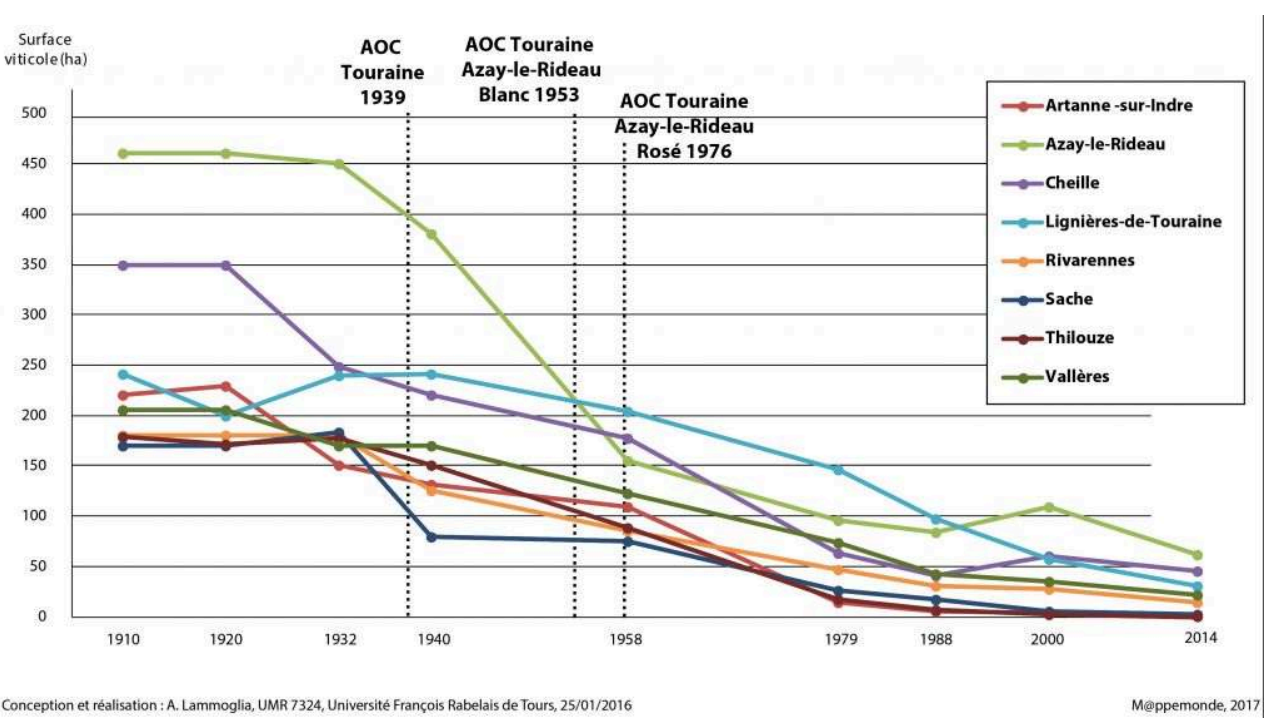




\section{Conclusion}

À l'issue de cette analyse, nous avons pu constater une permanence de l'activité viticole sur le territoire d'Azay-le-Rideau, démontrant une certaine résistance aux aléas de l'économie viticole (maladies de la vigne, changement de mode de consommation...). Une permanence, certes, mais aussi une dynamique spatiale importante et des mutations radicales en quatre siècles. Le vignoble ridellois a connu une phase d'expansion au XIX ${ }^{e}$ siècle, puis une phase de rétrécissement et de concentration aux $\mathrm{XX}^{\mathrm{e}}-\mathrm{XXI}^{\mathrm{e}}$ siècles sur quelques parcelles qui ne sont pas forcément localisées sur les sols les plus propices. Un exemple intéressant de ces mutations est celui du domaine de l'Aulée. En dépit de sa relative jeunesse (création dans le courant du XIX ${ }^{e}$ siècle sur des terrains peu favorables à la viticulture et qui n'étaient pas plantés jusqu'alors), ce domaine détient aujourd'hui une part considérable du vignoble ridellois. Même si nous n'avons pu approfondir ce cas particulier, il apparaît que cette présence de vigne est davantage liée au développement de structures professionnelles permettant une valorisation commerciale qu'à la qualité des sols.

Par ailleurs, l'article met en évidence des influences variables selon les différents facteurs. Le relief joue un rôle assez grand dans la localisation de la vigne (vignoble principalement de faible pente). Néanmoins, vu la diversité des sols sur lesquels la vigne a été plantée au cours des quatre siècles, la nature du sol ne semble pas être un facteur limitant. Nous avons aussi constaté que l'influence des facteurs pouvait évoluer au cours du temps et en fonction des contextes socio-économiques. La démographie en est le parfait exemple. Jusque dans les années 1880, nous avons constaté une corrélation très forte entre le nombre d'habitants et les superficies encépagées (faisant référence à un système d'autoconsommation). Cette corrélation s'est complètement inversée au cours $\mathrm{du} \mathrm{XX}^{\mathrm{e}}$ siècle, certainement en raison de la baisse de la consommation de vin et des changements de mode d'approvisionnement (marché en circuit long). En efFet, nous savons que les quantités de vin consommées annuellement en France ont fortement fluctué selon les époques, passant de 80 litres par habitant et par an au début du XIX ${ }^{\mathrm{e}}$ siècle à 160 litres un siècle plus tard (Argod-Dutard, Charvet et Lavaud, 2007, p. 723). Depuis, cette consommation a diminué de manière constante, n'étant plus aujourd'hui que d'une quarantaine de litres par habitant et par an (FranceAgriMer, 2012). Par ailleurs le mode d'approvisionnement a connu une véritable révolution, passant progressivement d'une société du circuit court avec une prévalence de l'autoconsommation à une société de consommation basée sur des approvisionnements lointains grâce au développement de moyens de transport rapides et peu coûteux (Argod-Dutard, Charvet et Lavaud 2007, p. 721). La combinaison de ces deux facteurs intervient sans aucun doute dans la décorrélation que l'on perçoit nettement dès les années 1930 à Azay-le-Rideau. Ainsi, la croissance démographique ridelloise, quoique relativement forte, ne provoque pas un accroissement du vignoble d'Azay-le-Rideau ni des communes voisines, parce que les Ridellois ne consomment plus guère les vins du cru, préférant acquérir des vins d'origine plus lointaine (circuit long).

Enfin, l'urbanisation et la labellisation ne semblent pas avoir joué un rôle majeur dans l'évolution du vignoble. L'étude montre qu'il y a une faible compétition spatiale à Azayle-Rideau entre l'urbain et la vigne, alors même que les surfaces bâties augmentent

Mappemonde, 120 | 2017 
fortement, en lien avec la croissance démographique. Mais à l'échelle du département, cette commune n'est pas un pôle démographique majeur, restant assez éloignée de l'agglomération tourangelle qui a connu, quant à elle, une forte croissance au $\mathrm{XX}^{\mathrm{e}}$ siècle. À titre d'exemple, Joué-les-Tours (commune limitrophe au sud de Tours) possédait un vignoble anciennement étendu et réputé au XIX ${ }^{\mathrm{e}}$ siècle; aujourd'hui la vigne y a complètement disparu alors que la population a été multipliée par 5 entre 1950 et 2000. L'éloignement d'Azay-le-Rideau au pôle urbain a donc probablement permis une croissance urbaine modérée, afFectant le vignoble ridellois de manière marginale. Concernant la labellisation, elle ne renverse pas la tendance générale à la réduction du vignoble, mais il est possible, sans qu'on puisse le démontrer, qu'elle soit propice au maintien des parcelles délimitées. Le tableau ci-dessous propose une synthèse de ces observations ainsi que nos hypothèses quant au poids des différents facteurs.

Tableau 3. Synthèse des observations et hypothèse quant au poids des facteurs

\begin{tabular}{|c|c|c|}
\hline Facteurs & Constat & Poids du facteur \\
\hline Relief & $\begin{array}{l}\text { Vignes installées préférentiellement sur de } \\
\text { faibles pentes, mais pas uniquement. }\end{array}$ & $\begin{array}{l}\text { Assez fort, mais ce n'est pas un } \\
\text { facteur limitant. }\end{array}$ \\
\hline $\begin{array}{l}\text { Nature du } \\
\text { sol }\end{array}$ & $\begin{array}{l}\text { Vignes installées préférentiellement sur des } \\
\text { sols favorables au XIX }{ }^{\mathrm{e}} \text { siècle, mais avec une } \\
\text { grande part du vignoble installée sur des sols } \\
\text { peu favorables en } 2014 \text {. }\end{array}$ & Assez faible \\
\hline Démographie & $\begin{array}{l}\text { Deux phases de corrélation entre la vigne et la } \\
\text { population: } \\
\text { - Avant } 1880 \text { : le développement de la vigne } \\
\text { suit le rythme de croissance démographique } \\
\text { (relation positive); } \\
\text { - À partir des années } 1930 \text { : on observe une } \\
\text { forte croissance démographique et une forte } \\
\text { décroissance viticole (relation négative). }\end{array}$ & $\begin{array}{l}\text { Fort : société } \\
\text { d'autoconsommation et marché en } \\
\text { circuit court } \\
\text { - Faible: la croissance } \\
\text { démographique n'entraîne pas } \\
\text { directement une diminution du } \\
\text { vignoble }\end{array}$ \\
\hline Urbanisation & $\begin{array}{l}\text { La décroissance du vignoble se fait } \\
\text { parallèlement à un accroissement de la } \\
\text { population et donc du bâti. Pour autant la } \\
\text { compétition spatiale semble marginale. }\end{array}$ & Faible \\
\hline Labellisation & $\begin{array}{l}\text { La seule labellisation n'a pas réussi à changer } \\
\text { la tendance à la décroissance du vignoble }\end{array}$ & Faible \\
\hline
\end{tabular}

In fine, si cette étude nous aide à comprendre et à évaluer le poids des difFérents facteurs, on s'aperçoit qu'aucun des cinq facteurs ne semble jouer un rôle déterminant et suffisant pour comprendre la dynamique complète du vignoble. Une analyse des facteurs économiques et socioprofessionnels permettrait sans doute d'afFiner la compréhension de la dynamique du vignoble ridellois. Un travail en cours a justement 
pour objectif d'analyser en profondeur la relation entre l'évolution de la consommation et celle des superficies viticoles en Touraine.

\section{BIBLIOGRAPHIE}

ARGOD-DUTARD F., CHARVET P., LAVAUD S. (2007). Voyage aux pays du vin : des origines à nos jours.

Histoire, anthologie, dictionnaire. Paris : R. LaFFont, 1312 p. ISBN 978-2221101421

DION R. (1959). Histoire de la vigne et du vin en France des origines au XIX ${ }^{e}$ siècle. Paris : CNRS Éditions, $772 \mathrm{p}$.

FERREIRA J. (2009). Le vignoble d'Azay-le-Rideau du XVII ${ }^{e}$ au XIX $X^{e}$ siècle. Mémoire de master 2 d'histoire, Tours, Université François-Rabelais.

FERREIRA J., LETURCQ S., MARTINEZ VASQUEZ L., VILLARS VILLECROZE B. de (2014). « Les dynamiques d'un terroir viticole Azay-le-Rideau, $\mathrm{XV}^{\mathrm{e}}-\mathrm{XIX}{ }^{\mathrm{e}}$ siècles ». In MARACHE C., LACHAUD S. ET BODINIER B. (éd.), L'Univers du vin. Hommes, paysages et territoires, Presses universitaires de Rennes, coll. «Bibliothèque d'Histoire Rurale », p. 203-220.

FRANCEAGRIMER (2012). « Les chifFres de la filière viti-vinicole ». Données et bilans, Vin, MontreuilSous-Bois, FranceAgriMer.

GARRIER G. (1989). Le Phylloxéra. Une guerre de trente ans, 1870-1900. Paris : Albin Michel, 194 p. ISBN 2-226-03879-5

GORRY J.-M. (2007). Paroisses et communes de France : dictionnaire d'histoire administrative et démographique. 37, Indre-et-Loire, Paris : CNRS Éditions, 479 p.

KUHNHOLTZ-LORDAT G. (1963). La genèse des appellations d'origine des vins. Mâcon : Imprimerie BuguetComptour, $150 \mathrm{p}$.

LETURCQ S. (2012). « De l'usage de la méthode régressive en Histoire médiévale ». Ménestrel. http:// www.menestrel.fr/?-methode-regressive-

TESSIER A. (2010). Le phylloxéra en Touraine. Mémoire de master 2 d'histoire, Tours, Université François-Rabelais.

\section{NOTES}

1. Les propriétaires actuels ne conservent aucune archive concernant l'histoire du domaine de l'Aulée. Les seules informations disponibles proviennent d'une enquête orale. Selon $\mathrm{M}^{\text {me }}$ Henrion, propriétaire de l'Aulée, le domaine aurait été fondé en 1856 par la famille Cordier qui l'a conservé jusqu'en 1946. Plusieurs propriétaires se succèdent ensuite jusqu'à la prise de contrôle du domaine en 1972 par la famille Lallier, apparentée à la famille champenoise Deutz. 


\section{RÉSUMÉS}

La dynamique spatiale des vignobles dans le temps long est régie par une multitude de facteurs en interaction. Dans le cadre de cette étude, ciblée sur le vignoble d'Azay-le-Rideau pour la période $\mathrm{XVII}^{\mathrm{e}}-\mathrm{XXI}^{\mathrm{e}}$ siècles, nous disposons de données permettant d'analyser cinq facteurs : la topographie, la nature du sol, la démographie, l'urbanisation et l'impact de la labellisation AOC. Une première partie, dédiée à l'analyse approfondie des mutations spatiales sur quatre siècles, met en évidence une certaine résistance de la vigne, avec toutefois des phases de forte croissance et décroissance, et un déplacement de la vigne sur le territoire communal. Dans une deuxième partie, nous étudions l'impact de ces cinq facteurs sur la dynamique globale du vignoble. Nous montrons qu'ils n'ont pas le même efret sur le développement de la vigne et que leur influence peut varier d'une époque à l'autre. Nous concluons en proposant une synthèse des observations, ainsi que des hypothèses quant au poids des différents facteurs. Dans le cadre du programme régional VitiTerroir, l'objectif, in fine, est de multiplier les études et d'intégrer les difFérents facteurs analysés dans un modèle multi-agent pour simuler la dynamique des vignobles ligériens à plusieurs échelles spatiales et temporelles.

The long term-spatial dynamics of vineyards is influenced by a multitude of interacting factors. This study focuses on the Azay-le-Rideau vineyard from the $17^{\text {th }}$ to the $21^{\text {st }}$ centuries. We have data that allows us to examine five factors: topography, soil characteristics, demography, urbanization, and the impact of the AOC label. The first section analyses the profound spatial mutations that have taken place over four centuries. It reveals that the vineyards are relatively resistant despite cyclical periods of strong growth and equally sharp decay; they have also shifted to communal territories. The second section focuses on the impact of these five factors on the global dynamics of the vineyard. We demonstrate that they do not equally influence the development of vineyards and their influence varies from one era to another. We conclude with a summary of observations along with hypotheses on the weight of the different factors. As part of the regional program, VitiTerroir, the ultimate goal is to multiply studies and integrate different factors analysed in a multi-agent model to simulate the dynamics of the regional vineyards at di FFerent temporal and spatial scales.

Las dinámicas espaciales que se producen en el territorio vitivinícola en el largo plazo están afectadas por una multitud de factores que interacción entre sí. Esta investigación, que se circunscribe al estudio del viñedo d'Azay-le-Rideau entre los siglos XVII al XXI, dispone de información que va a permitir analizar cinco factores : la topografía, las características del suelo, la demografía, la urbanización y el impacto de la denominación de origen. En una primera fase se analiza con profundidad las mutaciones espaciales producidas en estos cuatro siglos, evidenciando, con distintas fases de crecimiento o declive, una cierta pervivencia de la vid y su desplazamiento dentro del espacio comunal. En una segunda fase se estudia el impacto de estos cinco factores sobre la dinámica global de la viña. Estos no han tenido las mismas consecuencias en el desarrollo del viñedo, y su influencia ha podido variar de una época a otra, proponiéndose una síntesis de observaciones y de hipótesis según el peso de los distintos factores. En el marco del programa regional VitiTerroir, se quiere multiplicar los estudios e integrar los diferentes factores de análisis en un modelo multiagente para simular la dinámica del viñedo del País del Loira en distintas escalas espacio-temporales. 
INDEX

Mots-clés : Azay-le-Rideau, dynamiques spatiales, facteurs d'évolution, temps long (XVIIe-XXIe s.), territoire viticole

Palabras claves : Azay-le-Rideau, factores evolutivos, dinámicas espaciales, largo plazo (siglos XVII-XXI), territorios vitivinícolas

Keywords : Azay-le-Rideau, factors of evolution, spatial dynamics, long-term (17th-21st centuries), wine making territories

\section{AUTEURS}

\section{ADRIEN LAMMOGLIA}

Laboratoire Archéologie et Territoires - UMR 7324 CITERES, Université François-Rabelais de Tours

\section{SAMUEL LETURCQ}

Laboratoire Archéologie et Territoires - UMR 7324 CITERES, Université François-Rabelais de Tours 